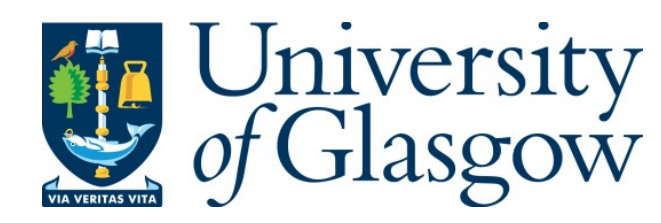

He, C., Zhang, L., Mao, J., Cao, A., Xiao, P., and Imran, M. A. (2016) Output SNR Analysis and Detection Criteria for Optimum DCT-Based Multicarrier System. In: 2016 International Symposium on Wireless Communications Systems (ISWCS), Poznań, Poland, 20-23 Sep 2016, pp. 59-64. ISBN 9781509020614.

There may be differences between this version and the published version. You are advised to consult the publisher's version if you wish to cite from it.

http://eprints.gla.ac.uk/132995/

Deposited on: 19 December 2016

Enlighten - Research publications by members of the University of Glasgow http://eprints.gla.ac.uk 


\title{
Output SNR Analysis and Detection Criteria for Optimum DCT-Based Multicarrier System
}

\author{
Chang He, Lei Zhang, Juquan Mao, Aijun Cao, Pei Xiao and Muhammad Ali Imran \\ Institute for Communication Systems, University of Surrey, United Kingdom, GU2 7XH \\ ZTE Wistron Telecom AB, Stockholm, Sweden, 16451 Kista \\ Emails:\{c.he, lei.zhang, juquan.mao, p.xiao,m.imran\}@surrey.ac.uk, cao.aijun@zte.com.cn
}

\begin{abstract}
The discrete cosine transform (DCT) based multicarrier system is regarded as one of the complementary multicarrier transmission techniques for 5th Generation (5G) applications in near future. By employing cosine basis as orthogonal functions for multiplexing each real-valued symbol with symbol period of $T$, it is able to reduce the minimum orthogonal frequency spacing to $1 /(2 T) \mathbf{H z}$, which is only half of that compared to discrete Fourier transform (DFT) based multicarrier systems. Critical to the optimal DCT-based system design that achieves interference-free single-tap equalization, not only both prefix and suffix are needed as symmetric extension of information block, but also a so-called front-end pre-filter is necessarily introduced at the receiver side. Since the pre-filtering process is essentially a time reversed convolution for continuous inputs, the output signal-to-noise ratio (SNR) for each subcarrier after filtering is enhanced. In this paper, the impact of pre-filtering on the system performance is analyzed in terms of ergodic output SNR per subcarrier. This is followed by reformulated detection criterion where such filtering process is taken into consideration. Numerical results show that under modified detection criteria, the proposed detection algorithms improve the overall bit error rate (BER) performance effectively.
\end{abstract}

\section{INTRODUCTION}

In addition to waveforms such as orthogonal frequency division multiplexing (OFDM) [1], [2], universal filtered multicarrier (UFMC) [3], [4], filtered OFDM [5], filter bank multicarrier (FBMC) [6], [7], [8], the discrete cosine transform (DCT) based multicarrier modulation (MCM) adopts cosinusoidal orthogonal functions $\cos (2 \pi \times k t /(2 T))$ instead of complex exponential functions set $\exp (j \times 2 \pi \times k t / T)$ to achieve minimum subcarrier spacing at $1 /(2 T) \mathrm{Hz}$ [9], where $k$ is the sub-channel index and $T$ is the symbol duration respectively. Correspondingly, the multiplexing and de-multiplexing of subcarriers can be simply implemented by inverse discrete cosine transform (IDCT) and DCT, instead of inverse discrete Fourier transform (IDFT) and DFT. The fast DCT algorithms proposed in [10] and [11] could provide fewer computational steps than FFT algorithms. This brings the advantage of reducing the signal-processing complexity and power consumption. Additionally, inter-carrier interference (ICI) coefficients in DCTMCM are more concentrated around the main coefficient than in DFT-MCM, leading to improved robustness against frequency offsets [12]. Since only one dimensional modulation is used for DCT-MCM the phase estimation in the coherent detection at the receiver is also simplified [13].

However, one of the major challenges that DCT-MCM encounters in practical design is that the DCT pair used for subcarrier multiplexing/demultiplexing does not have the circular convolution property under multipath channels as the cyclic prefix (CP) based DFT does in conventional systems [13], [14]. The DCT convolution property has been studied in the classical work in [15], which proves the fact that the DCT of the convolution output between two symmetrically extended sequences equals to the product of their individual DCTs. This requires the channel impulse response (CIR) to be symmetric inherently [13], [14]. But in more generic cases, such as multipath fading in wireless channels, the condition for symmetric convolution is not satisfied and the channel cannot be easily compensated by simple single-tap equalizer.

In the literature, there are various studies to address this problem. The chromatic dispersion in single-mode fibres (SMFs) [16] is one of the special channels which meet the symmetry condition, but it is only applicable under optical communication circumstance. The method proposed in [17] extends the DCT processed signal symmetrically to avoid the needs for channel symmetry. However, the net data rate is reduced by half. Another suboptimal method is based on a lowcomplexity detection algorithm [14], by which guard sequence is zero-padded and the DCT at the receiver is replaced by the DFT at doubled length. But there is still residual ICI which degrades overall performance. Besides the aforementioned design methods, a more optimized solution is given by AlDhahir in [13]. It enables single-tap equalization in the cosine domain without interference problem by using a time-domain finite impulse response (FIR) pre-filter at the receiver to filter CIR symmetric.

In this paper, we follow the optimal solution that introduces an additional pre-filter to ensure diagonalizable property by the DCT. In addition, both prefix and suffix that symmetrically extend the information sequence are inserted into each data block as guard sequence. Since the pre-filter is essentially a time-reverse filter and its coefficients in time domain are correlated with channel taps [13], the resulted overall effective channel coefficients become amplified, leading to increased signal power at the receiver. On the other hand, the filtering process renders noise a product of Gaussian variables, which no longer follows Gaussian distribution. As a consequence, the filtering effect needs to be taken into consideration to reformulate conventional detection criterion.

To the best of our knowledge, current work related to DCT- 

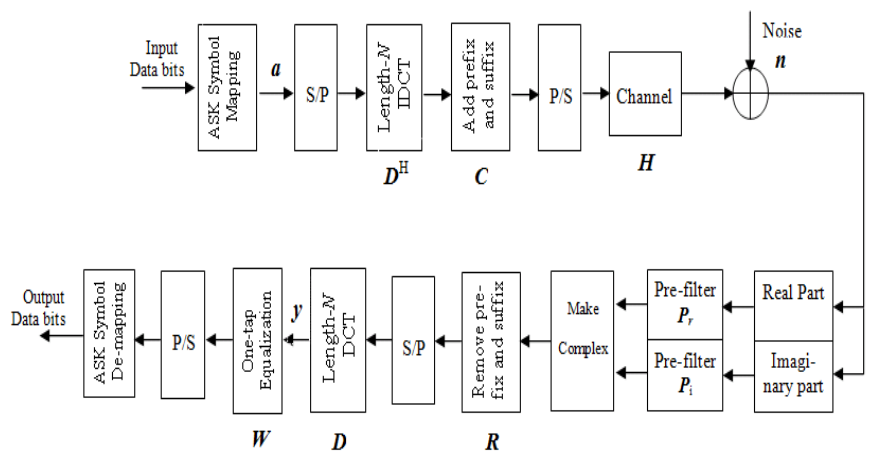

Fig. 1. Baseband equivalent model of optimum DCT-MCM system

based multicarrier system mostly discusses the corresponding properties and system implementation methods, e.g., in [13], [15]. The more in-depth study, such as how output signal-tonoise ratio (SNR) variates among subcarriers by the filtering process in DCT-MCM, however, has not been investigated. As a main contribution in this paper, we derive the relation between ergodic output SNR and input SNR on each subcarrier. Corresponding detection criterion is reformulated based on output instantaneous SNR to enhance overall system performance.

The remainder of the paper is organized as follows. We start in Section II by describing the transmission model of this optimum DCT-MCM system. This is followed by a mathematical formulation of the DCT optimal conditions for frequency selective channels. Section III shows analytically how the filtering process affects the ergodic output SNR and compares the SNR gain achieved under several practical channels. Modified detection criteria are then proposed for the minimummean-square-error (MMSE) and maximum likelihood (ML) methods by considering effective channel characteristics and noise filtering effect in Section IV, and the paper is concluded in Section V.

Notations: $[\cdot]^{H}$ and $[\cdot]^{T}$ stand for hermitian conjugate and transpose operation, respectively. $E[\cdot]$ is defined as the expectation of random variable. We use $\operatorname{diag}(\cdot)$ to return the vector of the main diagonal elements of operated matrix. $\mathbf{I}_{N}$ and $\mathbf{J}_{N}$ refer to identity matrix and reversal matrix of dimension $N$, respectively. $\mathbf{0}_{M \times N}$ is the zero matrix of size $M \times N$. A linear convolution operation of two vectors is denoted as $*$.

\section{SySTEM DESCRIPTION}

In this section, we first explain a baseband model of optimum DCT-MCM system over the wireless frequency-selective channel which is illustrated in Fig. 1 [13]. All processing steps are interpreted by matrix operations, paving the road to further system performance analysis. As can be seen from the figure, the DCT/IDCT pair is employed for modulation/demodulation whilst a pre-filter is necessarily imposed on the front of the receiver to guarantee channel symmetry condition.

Assume $N$ subcarriers are employed for carrying information data streams, and $N$ ASK symbols are imposed on those $N$ subcarriers before transmission. It is noted in the figure that the real and imaginary part of the received symbols are processed separately and their corresponding processing steps are identical. For simplicity, we express the general system model using only real branch of the diagram by

$$
\mathbf{y}=\mathbf{D R} \mathbf{P}_{r} \mathbf{H}_{r} \mathbf{C D}^{H} \mathbf{a}+\mathbf{D R} \mathbf{P}_{r} \mathbf{n}_{r}
$$

where $\mathbf{y}$ is the signal received as the input to the equalizer, $\mathbf{a} \in$ $\mathbb{R}^{N \times 1}$ is an uncorrelated ASK symbol vector with zero mean and normalized power $\sigma_{s}^{2}$. $\mathbf{D} \in \mathbb{R}^{N \times N}$ is power normalized DCT matrix in type II which is the most popular member of the DCT family [15]. $\mathbf{C} \in \mathbb{R}^{\left(N+L_{p}+L_{s}\right) \times N}$ is the matrix implementation of adding prefix (length $L_{p}$ ) and suffix (length $L_{s}$ ) in symmetric form defined as

$$
\mathbf{C}=\left[\mathbf{J}_{L_{p}}, \mathbf{0}_{L_{p} \times\left(N-L_{p}\right)} ; \mathbf{I}_{N} ; \mathbf{0}_{L_{s} \times\left(N-L_{s}\right)}, \mathbf{J}_{L_{s}}\right]
$$

Generally, the prefix and suffix are assigned to be identical to the guard sequence length $\left(L_{s}=L_{p}\right)$ and the total block length $L_{1}$ is given by $L_{1}=N+L_{p}+L_{s}$. Assuming $\mathbf{h}=\left[h_{1}, h_{2}, \ldots, h_{L}\right]$ is the real component of channel impulse response with length $L$, the corresponding channel convolution matrix denoted as $\mathbf{H}_{r} \in \mathbb{R}^{L_{1} \times L_{1}}$ is organised as a Toeplitz matrix with the first row and first column being $\left[h_{L}, h_{L-1}, \ldots, h_{1}, \mathbf{0}_{1 \times\left(L_{1}-L\right)}\right]$ and $\left[h_{L}, \mathbf{0}_{1 \times\left(L_{1}-1\right)}\right]^{T}$ respectively. For simplicity, we define $\mathbf{g}=\left[g_{1}, g_{2}, \ldots, g_{L}\right]=$ $\left[h_{L}, h_{L-1}, \ldots, h_{1}\right]$. In order to combat multipath effect, sufficient guard sequence should be guaranteed $\left(L \leq L_{s}=\right.$ $L_{p}$ ) [13]. On the other hand, the pre-filter matrix $\mathbf{P}_{r} \in$ $\mathbb{R}^{L_{1} \times L_{1}}$ representing time-reverse filtering is also implemented by a Toeplitz matrix with its first row and column being $\left[h_{L}, \mathbf{0}_{1 \times\left(L_{1}-1\right)}\right]$ and $\left[\mathbf{g}, \mathbf{0}_{1 \times\left(L_{1}-L\right)}\right]^{T}$, respectively. $\mathbf{R} \in$ $\mathbb{R}^{N \times L_{1}}$ is the matrix representation of the prefix and suffix removal operation before demultiplexing, which can be defined as

$$
\mathbf{R}=\left[\mathbf{0}_{N \times L_{p}}, \mathbf{I}_{N}, \mathbf{0}_{N \times L_{s}}\right] .
$$

By adding the pre-filter and designing the prefix and suffix guard sequences according to the aforementioned method, it ensures optimality of the DCT for multicarrier transmission in the sense that both ICI and inter-symbol interference (ISI) are completely eliminated. Furthermore, based on the theorem in [13], the resultant output matrix $\mathbf{D R P}_{r} \mathbf{H}_{r} \mathbf{C D}^{H}$ is equivalent to a diagonal matrix which can be written as

$$
\mathbf{H}_{e f f, r}=\mathbf{D R P}_{r} \mathbf{H}_{r} \mathbf{C D}^{H}
$$

where $\mathbf{H}_{e f f, r} \in \mathbb{R}^{L_{1} \times L_{1}}$ is the equivalent channel matrix for real part branch with its diagonal vector defined by

$$
\operatorname{diag}\left(\mathbf{H}_{e f f, r}\right)=\left[H_{1}, H_{2}, \ldots, H_{N}\right]
$$

In DFT-MCM systems, channel frequency responses are assumed to follow the Gaussian distribution and with zero mean and same variance on each subcarrier if Rayleigh channel is considered. However, this is not the case in DCTMCM systems as the received signal power is not identically distributed among subcarriers after correlated filtering. The corresponding effective frequency responses no longer follow the Gaussian distribution. This requires further analysis on output SNR which is described in the following section. 


\section{OUtPut SnR AnAlysis}

In order to analyze the filtering effect on DCT-MCM for general multipath channels, the expressions for ergodic output signal power and ergodic output noise power are derived respectively in this section. Note that we consider an ASK modulated system in which real symbols pass through a complex channel. The received outputs are complex correspondingly that can be viewed as two streams correlated by channel in quadrature, with each allocating one-half the total received power. The pre-filters are then employed to filter both its real and imaginary data stream independently as indicated in Fig. 1. As to detection, the two filtered streams are then combined in the complex domain for equalization. Since the two streams experience identical processing steps, for simplicity, the following derivations are for the real branch and total power is thus can be calculated by doubling the result.

\section{A. Ergodic Output Signal Power}

The channel symmetry condition can be met in practice by time-reversed filtering, resulting in the overall symmetric channel obtained by

$$
\begin{aligned}
\mathbf{f} & =\mathbf{h} * \mathbf{g}=\left[h_{1}, h_{2}, \ldots, h_{L}\right] *\left[h_{L}, h_{L-1}, \ldots, h_{1}\right] \\
& =\left[f_{1}, f_{2}, \ldots, f_{L}, \ldots, f_{2 L-1}\right]
\end{aligned}
$$

where $\mathbf{f}=\left[f_{1}, f_{2}, \ldots, f_{L}, \ldots, f_{2 L-1}\right]$ is a symmetric channel impulse response of length $2 L-1$. The channel-coefficients $f_{i}$ are denoted by

$$
f_{i}=\left\{\begin{array}{lr}
\sum_{k=1}^{i} h_{k} h_{L-i+k} & \text { if } 1 \leq i \leq L \\
\sum_{k=1}^{2 \bar{L}-i} h_{k} h_{i-L+k} & \text { if } L<i \leq 2 L-1 .
\end{array}\right.
$$

The symmetry property can be verified by the condition $f_{i}=$ $f_{2 L-i}$ as indicated in Eq. (7).

Lemma 1: All matrices diagonalizable by the type-II DCT matrix can be written as the sum of an $N \times N$ symmetric Toeplitz matrix $\mathbf{H}_{T}$ and an $N \times N$ Hankel matrix $\mathbf{H}_{H}$; i.e., $\mathbf{D}\left(\mathbf{H}_{T}+\mathbf{H}_{H}\right) \mathbf{D}^{H}=\mathbf{H}_{e f f, r}[13]$.

In this case, $\mathbf{H}_{T}$ is a Toeplitz matrix derived by the first row and first column being $\mathbf{z}=$ $\left[z_{1}, z_{2}, \ldots, z_{N}\right]=\left[f_{L}, f_{L-1}, \ldots, f_{1}, \mathbf{0}_{1 \times(N-L)}\right]$ and $\left[f_{L}, f_{L-1}, \ldots, f_{1}, \mathbf{0}_{1 \times(N-L)}\right]^{T}$ while $\mathbf{H}_{H}$ is a Hankel matrix derived by the first row and first column being $\left[f_{L-1}, f_{L-2}, \ldots, f_{1}, \mathbf{0}_{1 \times(N-L+1)}\right]$ and $\left[f_{L-1}, f_{L-2}, \ldots, f_{1}, \mathbf{0}_{1 \times(N-L+1)}\right]^{T}$ respectively. By properly designing the guard sequence and the pre-filter, the integrated matrix $\mathbf{R P}_{r} \mathbf{H}_{r} \mathbf{C}$ becomes diagonalizable by the type-II DCT matrix. From the Lemma 1, we yield

$$
\mathbf{R} \mathbf{P}_{r} \mathbf{H}_{r} \mathbf{C}=\mathbf{H}_{T}+\mathbf{H}_{H}
$$

The theorem given in [15] indicates all $N \times N$ matrices diagonalizable by the type-II DCT matrix can be generated with the type-I DCT matrix as

$$
\mathbf{D}\left(\mathbf{H}_{T}+\mathbf{H}_{H}\right) \mathbf{D}^{H}=\mathbf{D}_{I} \mathbf{z}=\mathbf{H}_{e f f, r}
$$

where $\mathbf{D}_{I}$ represents the DCT type-I matrix. As a consequence, the main diagonal vector coefficients of $\mathbf{H}_{e f f, r}$ in Eq. (5) can be derived by DCT-I with vector $\mathbf{z}$, which is represented as follow

$$
\begin{aligned}
H_{k}= & z_{1}+(-1)^{k-1} \cdot z_{N} \\
& +2 \sum_{n=2}^{N-1} z_{n} \cdot \cos \left[\frac{\pi}{N}(n-1)(k-1)\right]
\end{aligned}
$$

On the other hand, the real component of channel coefficients is Gaussian distributed and can be denoted as $h_{k} \sim$ $N\left(0,0.5 \sigma_{k}^{2}\right)$. Since the channel is power normalised, we have

$$
\sum_{k=1}^{L} \sigma_{k}^{2}=1
$$

By using the assumption that different channel taps are uncorrelated, we have

$$
E\left[h_{i} \cdot h_{j}\right]=0 \quad \text { if } i \neq j
$$

By combining Eq.(7) and Eq.(11), we can obtain the expected correlation value of effective channel coefficients as

$$
\begin{gathered}
E\left[f_{i} \cdot f_{j}\right]= \\
\left\{\begin{array}{cl}
0 & i \neq j ; \\
\frac{1}{4} \sum_{p=1}^{i} \sigma_{p}^{2} \sigma_{L-i+p}^{2} & i=j \neq L ;(13) \\
\frac{3}{4} \sum_{p=1}^{L} \sigma_{p}^{4}+\frac{1}{2} \sum_{p=1}^{L} \sum_{t=1}^{L-i} \sigma_{p}^{2} \sigma_{p+t}^{2} & i=j=L .
\end{array}\right.
\end{gathered}
$$

By applying Eq. (13) to Eq. (10), the ergodic output signal power of real branch for the $k$ th subcarrier is amplified as

$$
E\left[H_{k}^{2}\right]=E\left[f_{L}^{2}\right]+4 \sum_{n=2}^{L} E\left[f_{L-n+1}^{2}\right] \cdot \cos ^{2}\left[\frac{\pi}{N}(n-1)(k-1)\right]
$$

Apparently, the signal power is maximized if the multiplying factor $\cos ^{2}\left[\frac{\pi}{N}(n-1)(k-1)\right]$ equals to 1 , which is satisfied by the first subcarrier at $k=1$, i.e.,

$$
\begin{aligned}
E\left[H_{1}^{2}\right] & =E\left[f_{L}^{2}\right]+4 \sum_{n=2}^{L} E\left[f_{L-n+1}^{2}\right] \\
& =\frac{3}{4} \sum_{p=1}^{L} \sigma_{p}^{4}+\frac{3}{2} \sum_{p=1}^{L} \sum_{t=1}^{L-i} \sigma_{p}^{2} \sigma_{p+t}^{2}=0.75
\end{aligned}
$$

This implies the amplifying factor $E\left[H_{1}^{2}\right]$ is a constant value equals to 0.75 . Considering this is just for the real branch of the processed signal, which only accounts half of total power. The received average power on the first subcarrier is amplified by a factor of 1.5. The gain is less for other subcarriers, i.e., $2 E\left[H_{k}^{2}\right] \leq 2 E\left[H_{1}^{2}\right]$. From Eq.(15), it is clear to see that the gain varies for each subcarrier and we are able to calculate ergodic output signal power for each subcarrier theoretically.

\section{B. Ergodic Output Noise Power}

The output noise variance of the system is also changed due to the filtering operation. As mentioned in the last section, the information symbols could be detected by either the inphase or quadrature dimension. Practically, both the real and imaginary components of the complex baseband equivalent CIR are constrained to be symmetric by implementing a complex pre-filter. This is similar to two receiver diversity case. 
The received noise $\mathbf{v}$ consists of two independent components which can be represented as

$$
\mathbf{v}=\mathbf{D R}\left(\mathbf{P}_{r} \mathbf{n}_{r}+j \mathbf{P}_{i} \mathbf{n}_{i}\right)
$$

where $\mathbf{P}_{r}$ and $\mathbf{P}_{i}$ are the real and imaginary parts of the complex-valued pre-filter matrix $\mathbf{P}$; whereas $\mathbf{n}_{r}$ and $\mathbf{n}_{i}$ are the real and imaginary parts of the AWGN noise vector $\mathbf{n}$ with variance $\sigma_{n}^{2}$. The ergodic output noise covariance matrix $E\left[\mathbf{v} \mathbf{v}^{H}\right]$ can thus be expressed as follows

$$
\begin{aligned}
E\left[\mathbf{v v}^{H}\right] & =E\left[\mathbf{D R}\left(\mathbf{P}_{r} \mathbf{n}_{r}+j \mathbf{P}_{i} \mathbf{n}_{i}\right)\left(\mathbf{P}_{r} \mathbf{n}_{r}+j \mathbf{P}_{i} \mathbf{n}_{i}\right)^{H} \mathbf{R}^{H} \mathbf{D}^{H}\right] \\
& =E\left[\mathbf{D R}\left(\mathbf{P}_{r} \mathbf{n}_{r} \mathbf{n}_{r}^{H} \mathbf{P}_{r}^{H}+\mathbf{P}_{i} \mathbf{n}_{i} \mathbf{n}_{i}^{H} \mathbf{P}_{i}^{H}\right) \mathbf{R}^{H} \mathbf{D}^{H}\right]
\end{aligned}
$$

Since $E\left[\mathbf{n}_{r} \mathbf{n}_{r}^{H}\right]=E\left[\mathbf{n}_{i} \mathbf{n}_{i}^{H}\right]=\frac{\sigma_{n}^{2}}{2} \mathbf{I}$ and $E\left[\mathbf{P}_{r} \mathbf{P}_{r}^{H}\right]=E\left[\mathbf{P}_{i} \mathbf{P}_{i}^{H}\right]$, Eq. (17) can be simplified to

$$
\begin{aligned}
E\left[\mathbf{v v}^{H}\right] & =\frac{\sigma_{n}^{2}}{2} E\left[\mathbf{D R}\left(\mathbf{P}_{r} \mathbf{P}_{r}^{H}+\mathbf{P}_{i} \mathbf{P}_{i}^{H}\right) \mathbf{R}^{H} \mathbf{D}^{H}\right] \\
& =\sigma_{n}^{2} \mathbf{D R} E\left[\mathbf{P}_{r} \mathbf{P}_{r}^{H}\right] \mathbf{R}^{H} \mathbf{D}^{H}
\end{aligned}
$$

The matrix $\mathbf{P}_{r}$ consists of channel coefficients that satisfy Eq. (12), $E\left[\mathbf{P}_{r} \mathbf{P}_{r}^{H}\right]$ then becomes a diagonal matrix defined as $E\left[\mathbf{P}_{r} \mathbf{P}_{r}^{H}\right]=\mathbf{T}=\operatorname{diag}(\mathbf{t})$ where $\mathbf{t}$ is a vector of size $L_{1}$ and $\mathbf{t}=\left[t_{1}, t_{2}, \ldots, t_{L_{1}}\right]$. Convolving the two inputs, we summarize the results as

$$
t_{m}=\left\{\begin{array}{lr}
\sum_{i=1}^{m} h_{L-i+1}^{2} & \text { if } i \leq m \leq L ; \\
\sum_{i=1}^{L} h_{L-i+1}^{2}=0.5 & \text { if } L<m \leq L_{1} .
\end{array}\right.
$$

Substituting Eq. (19) to Eq. (18), we obtain

$$
E\left[\mathbf{v v}^{H}\right]=0.5 \sigma_{n}^{2} \quad \text { if } L \leq L_{s}\left(\text { or } L_{p}\right)
$$

which is a constant value. This reveals the fact that by prefiltering the in-phase and quadrature branches independently, the overall noise power at the output is reduced by half if sufficient guard sequence length is guaranteed as indicated in Eq. (20).

\section{Gain of ergodic output SNR}

With the derivations of output signal power and noise power provided, we can exam how much gain DCT-MCM is achieved in terms of SNR. Since the SNR loss due to guard interval can be easily incorporated if the length of guard interval and the DCT/FFT size are known, we neglect this effect in the following derivations. The input SNR is defined as

$$
S N R_{i n}=\frac{\sigma_{s}^{2}}{\sigma_{n}^{2}} \quad \text { for all subcarrier index } k
$$

On the other hand, the output SNR can be expressed by the previous results expressed in Eq. (14) and Eq. (20) as

$$
S N R_{\text {out }}(k)=\frac{E\left[H_{k}^{2}\right] \cdot \sigma_{s}^{2}}{0.5 \sigma_{n}^{2}}=2 E\left[H_{k}^{2}\right] \cdot S N R_{\text {in }}
$$

Consequently, the gain $\eta$ of SNR achieved at the output is

$$
\begin{aligned}
\eta(k)= & 2\left(E\left[f_{L}^{2}\right]+4 \sum_{n=2}^{L} E\left[f_{L-n+1}^{2}\right]\right. \\
& \left.\cdot \cos ^{2}\left[\frac{\pi}{N}(n-1)(k-1)\right]\right)
\end{aligned}
$$

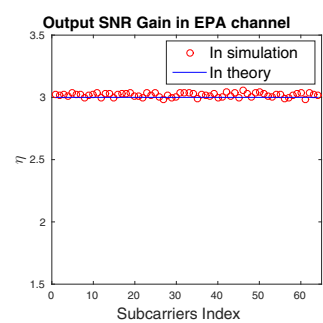

(a)

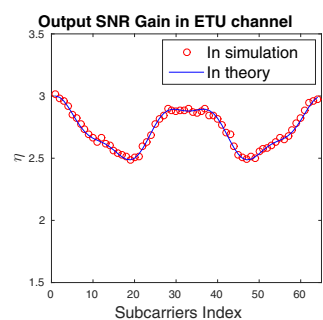

(c)

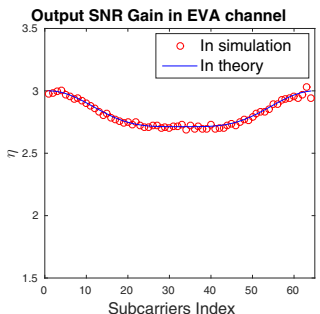

(b)

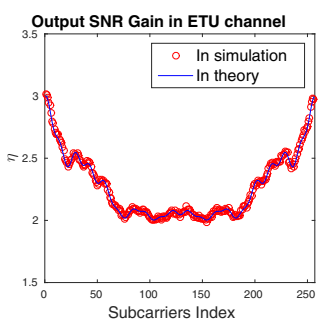

(d)
Fig. 2. Output SNR gain in different channels.

Eq.(23) implies the output SNR gain has very close relations with the subcarrier index and channel characteristics. We are now able to analyze the SNR gain among subcarriers. The theoretical analysis is validated by the means of MonteCarlo simulations. For comparison, three widely considered channels are employed namely, Extended Vehicular A model (EVA), Extended Typical Urban model (ETU) and Extended Pedestrian A model (EPA), respectively. The sampling rate is $f_{s}=20 \mathrm{MHz}$ and DCT size is assumed to be 64 and 256 .

Fig. 2 illustrates the simulation results for linear SNR gain at the output of the pre-filter among subcarriers for these three channels; theoretical results are also shown as comparison. One can see from the figure, that the simulation results coincide with the theoretic analysis in general. In the first three sub-figures, although the same subcarrier number is assumed for transmission through different channels, the corresponding curves demonstrate very distinct characteristics. Generally, the worse condition of the channel, the more frequency selectivity among subcarriers we have as a result of pre-filtering. Fig 2 . (a), in particular, achieves constant gain among all subcarriers. This is because EPA channel has a single tap if $f_{s}=20 \mathrm{MHz}$. If we focus on the ETU channel and increase the subcarrier number to 256, the second term in Eq. (23) will have more fluctuations as variable number increases along with subcarrier number, which is verified by Fig. 2. (d).

On the other hand, the output SNR gain varies among subcarriers. Most subcarriers see an enhanced output SNR by 2 to 3 folds. The above analysis will be utilized in the design of detection schemes in the equal.

\section{DETECTION CRITERION FOR DCT-MCM SYSTEM}

The commonly employed multi-carrier data detection techniques include linear methods such as zero forcing (ZF), minimum-mean-square-error (MMSE) detection, as well as the maximum likelihood (ML) detection that achieves optimal performances at the cost of high computational complexities [18]. The ZF one-tap equalizer only requires the knowledge 
of the channel and is the most straightforward to implement at the receiver [19]. On the other hand, the MMSE equalizer requires the knowledge of the noise variance in addition to the channel information. However, as analysed earlier, the filtered noise on each subcarrier becomes correlated with each other, therefore is no longer Gaussian distributed. Consequently, the detection criterion needs to be changed in DCT-MCM system. This motivates us to focus on reformulating the MMSE and ML criterion to make them applicable to DCT-MCM so as to bring performance improvement.

\section{A. MMSE Detection}

The MMSE detection aims to minimize the mean square error $E(\|\mathbf{W y}-\mathbf{a}\|)$ [19], [20]. Here $\|\cdot\|$ stands for Euclidean norm of a vector. To facilitate derivation, we represent the received signal in terms of effective real and imaginary channel matrix $\mathbf{H}_{e f f, r}$ and $\mathbf{H}_{e f f, i}$ combined with effective pre-filtering matrix $\mathbf{G}_{r}$ and $\mathbf{G}_{i}$ defined as

$$
\begin{aligned}
\mathbf{y} & =\left(\mathbf{H}_{e f f, r}+j \mathbf{H}_{e f f, i}\right) \mathbf{a}+\mathbf{G}_{r} \mathbf{n}_{r}+j \mathbf{G}_{i} \mathbf{n}_{i} \\
& =\mathbf{H}_{e f f} \mathbf{a}+\mathbf{G}_{r} \mathbf{n}_{r}+j \mathbf{G}_{i} \mathbf{n}_{i}
\end{aligned}
$$

Where $\mathbf{G}_{r}=\mathbf{D R} \mathbf{P}_{r}$ and $\mathbf{G}_{i}=\mathbf{D R} \mathbf{P}_{i} . \mathbf{H}_{e f f}$ is the complex effective channel matrix. In the $\mathrm{ZF}$ method, since the equalization matrix $\mathbf{W}_{Z F}=\mathbf{H}_{e f f}^{H} /\left|\mathbf{H}_{e f f}\right|^{2}$ does not depends on the noise characteristic, conventional detection criterion is still applicable to the DCT-MCM system. However, as can be seen from Eq. (24), the overall noise $\mathbf{G}_{r} \mathbf{n}_{r}+j \mathbf{G}_{i} \mathbf{n}_{i}$ in the DCT-MCM system is correlated by multiplying a nondiagonal matrix with both the real and imaginary component respectively. The MMSE detection considers the noise variance and mitigates the noise enhancement problem by using the minimum mean square error equalization matrix $\mathbf{W}_{M M S E}$ satisfying the following condition

$$
\frac{\partial}{\partial \mathbf{W}_{M M S E}} E\left[\left(\mathbf{W}_{M M S E} \mathbf{y}-\mathbf{a}\right)\left(\mathbf{W}_{M M S E} \mathbf{y}-\mathbf{a}\right)^{H}\right]=0
$$

Substituting Eq. (24) to Eq. (25), we yield

$\mathbf{W}_{M M S E}\left[\sigma_{s}^{2} \mathbf{H}_{e f f} \mathbf{H}_{e f f}^{H}+0.5 \sigma_{n}^{2}\left(\mathbf{G}_{r} \mathbf{G}_{r}^{H}+\mathbf{G}_{i} \mathbf{G}_{i}^{H}\right)\right]=\sigma_{s}^{2} \mathbf{H}_{e f f}^{H}$

By moving the multiplying component to the right of the equation, the reformulated expression of MMSE equalization matrix is given by

$$
\mathbf{W}_{M M S E}=\frac{\sigma_{s}^{2} \mathbf{H}_{e f f}^{H}}{\sigma_{s}^{2}\left|\mathbf{H}_{e f f}\right|^{2}+0.5 \sigma_{n}^{2}\left(\mathbf{G}_{r} \mathbf{G}_{r}^{H}+\mathbf{G}_{i} \mathbf{G}_{i}^{H}\right)}
$$

As evidenced by the above equation, unlike the conventional MMSE detection method, the knowledge of the pre-filters information $\mathbf{G}_{r}$ and $\mathbf{G}_{i}$ are necessarily required for the MMSE detection for DCT-MCM systems, as the effective noise after pre-filter would change instantaneous output SNR.

\section{B. Maximum Likelihood Detection}

Compared to linear detectors which experience degraded performance, the ML detection is a superior solution and achieves optimal performance. The received symbol on $k$ th subcarrier in DCT-MCM system is expressed from Eq. (1) as

$$
y_{k}=H_{k} a_{k}+\sum_{t=1}^{N} g_{k, t} n_{r, t}+j \sum_{t=1}^{N} p_{k, t} n_{i, t}
$$

Where $g_{k, t}$ and $p_{k, t}$ are the elements in $k$ th row and $t$ th column of $\mathbf{G}_{r}$ and $\mathbf{G}_{i}$ respectively; whereas $n_{r, t}$ and $n_{i, t}$ are respectively the real and imaginary parts of noise vector $\mathbf{n}$ on the $t$ th subcarrier. Generally, the coefficients $g_{k, t}$ and $p_{k, t}$ are time-reversed transforms from channel entries, rendering the integrated noise component in Eq. (28) in the form of a series of complex and correlated combinations which no longer follows Gaussian distribution in statistics.

In practice, the transmitted signals are divided into several frames with each frame sharing the same channel information for all the blocks. Consequently, the multiplying factors $g_{k, t}$ and $p_{k, t}$ are instantaneous identical constants for every block within one frame. Under this assumption, the integrated noise is regarded as multiple additions of arbitrary number of Gaussian noise variables with variance of $g_{k, t}^{2}$ on the real part and $p_{k, t}^{2}$ on the imaginary part. The overall variance $D_{0, k}$ is then calculated as

$$
D_{0, k}=\frac{N_{0}}{2}\left(\sum_{t=1}^{N} g_{k, t}^{2}+\sum_{t=1}^{N} p_{k, t}^{2}\right)
$$

Based on this instantaneous output noise as complex-Gaussian variable with variance $D_{0, k}$, the conditional probability density function of $y_{k}$ given $a_{k}$ is in the formula as $p\left(y_{k} \mid a_{k}\right)=$ $\frac{1}{\sqrt{\pi D_{0, k}}} \exp \left(-\frac{\left|y_{k}-H_{k} a_{k}\right|^{2}}{D_{0, k}}\right)$. Note that this holds for a specific data frame for which the $g_{k, t}$ and $p_{k, t}$ can be regarded as constant values. The soft information at the $i$ th bit is then expressed by the mean of log-likelihood ration (LLR) as [21]

$$
\begin{aligned}
L_{i} & =\ln \frac{\sum_{a_{k} \in \mathcal{A}_{i}^{(1)}} p\left(a_{k} \mid y_{k}\right)}{\sum_{a_{k} \in \mathcal{A}_{i}^{(0)}} p\left(a_{k} \mid y_{k}\right)}=\ln \frac{\sum_{a_{k} \in \mathcal{A}_{i}^{(1)}} p\left(y_{k} \mid a_{k}\right)}{\sum_{a_{k} \in \mathcal{A}_{i}^{(0)}} p\left(y_{k} \mid a_{k}\right)} \\
& =\ln \frac{\sum_{a_{k} \in \mathcal{A}_{i}^{(1)}} \exp \left(\frac{-\left|y_{k}-H_{k} a_{k}\right|^{2}}{D_{0, k}}\right)}{\sum_{a_{k} \in \mathcal{A}_{i}^{(0)}} \exp \left(\frac{-\left|y_{k}-H_{k} a_{k}\right|^{2}}{D_{0, k}}\right)}
\end{aligned}
$$

The Eq. (30) is complicated and can be simplified by log-sumexponential approximation [10]: $\log \sum_{i} \exp \left(\phi_{i}\right)=\max _{i}\left(\phi_{i}\right)$. By eliminating numerical stability and assuming $z_{k}=y_{k} / a_{k}$, the LLR equation can be rewritten as

$$
\begin{aligned}
L_{i} & \approx \ln \frac{\max _{a_{k} \in \mathcal{A}_{i}^{(1)}} \exp \left[-\left|H_{k}\right|^{2}\left(z_{k}-a_{k}\right)^{2} / D_{0, k}\right]}{\max _{a_{k} \in \mathcal{A}_{i}^{(0)}} \exp \left[-\left|H_{k}\right|^{2}\left(z_{k}-a_{k}\right)^{2} / D_{0, k}\right]} \\
& =\frac{D_{0, k}}{\left|H_{k}\right|^{2}}\left\{\max _{a_{k} \in \mathcal{A}_{i}^{(1)}}\left[-\left(z_{k}-a_{k}\right)^{2}\right]-\max _{a_{k} \in \mathcal{A}_{i}^{(0)}}\left[-\left(z_{k}-a_{k}\right)^{2}\right]\right\} \\
& =\frac{D_{0, k}}{\left|H_{k}\right|^{2}}\left\{-\min _{a_{k} \in \mathcal{A}_{i}^{(1)}}\left(z_{k}-a_{k}\right)^{2}+\min _{a_{k} \in \mathcal{A}_{i}^{(0)}}\left(z_{k}-a_{k}\right)^{2}\right\}(31)
\end{aligned}
$$

Similar to MMSE detection, the part $\frac{D_{0, k}}{\left.H_{k}\right|^{2}}$ in Eq. (31) implies the modified ML criterion also uses instantaneous output SNR by taking into account the noise filter effect. 


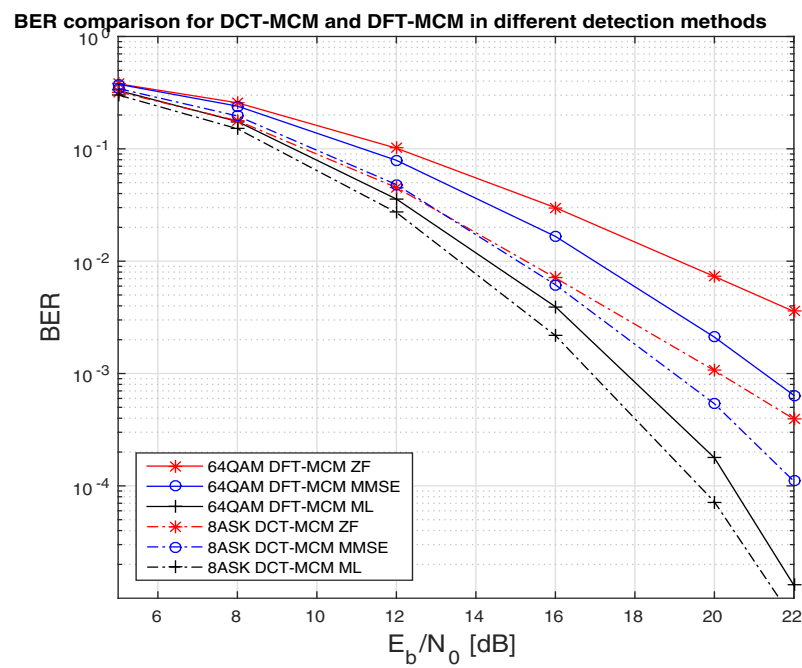

Fig. 3. BER comparison for DCT-MCM and DFT-MCM in different detection methods

\section{Comparison between $Z F, M M S E$ and $M L$ detections}

Based on the above reformulated detection criterion, we compare the BER performance in simulation between DCTMCM and DFT-MCM systems under a typical Wifi channel. Since the subcarrier spacing in DCT-MCM is half of that in DFT-MCM, we assign 128 subcarriers to DCT-MCM with 8ASK scheme and 64 subcarriers to DFT-MCM with 64 quadrature amplitude modulation (QAM) scheme respectively to keep the same bandwidth efficiency. A CP with length of 12 is introduced to DFT-MCM whereas DCT-MCM has a guard sequence of double length. In addition, the polynomial $(133,171)$ code with constraint length of 7 and rate of $1 / 2$ is employed for both systems. In the Fig. 3, DCT-MCM outperforms DFT-MCM on all accounts. The gap for ZF and MMSE between these two systems are about $4 \mathrm{~dB}$ and 2 $\mathrm{dB}$ respectively, showing clear superiority and very attractive advantage of DCT-MCM over DFT-MCM. However, this gain is reduced to around $1 \mathrm{~dB}$ if $\mathrm{ML}$ is employed for the two systems, which is illustrated in the figure.

\section{CONCLUSION}

In this paper, the gain of output SNR at the receiver of the DCT-MCM system is derived analytically for each subcarrier. It has been shown the filtering operation introduces channel correlation to the received signals, leading to a output SNR gain up to three folds. The gain depends on subcarrier index and channel characteristics. As to the detection techniques, we propose methods to take into account output instantaneous SNR, based upon which the criterion for MMSE and ML are reformulated respectively. The BER results verify the performance enhancement by considering filtering effect.

\section{REFERENCES}

[1] L. Wen, R. Razavi, M. A. Imran, and P. Xiao, "Design of joint sparse graph for OFDM system", IEEE Transactions on Wireless Communications, vol. 14, no. 4, pp. 1823-1836, April 2015.

[2] A. Ijaz, L. Zhang, M. Grau, A. Mohamed, S. Vural, A. Quddus, M. Imran, C. H. Foh, and R. Tafazolli, "Enabling massive iot in 5G and beyond systems: PHY radio frame design considerations", IEEE Access, 2016.

[3] L. Zhang, P. Xiao, and A. Quddus, "Cyclic prefix based universal filtered multi-carrier system and performance analysis", IEEE Signal Processing Letters, 2016.

[4] L. Zhang, A.Ijaz, P. Xiao, A. Quddus, and R. Tafazolli, "Single-rate and multi-rate multi-service systems for next generation and beyond communications", IEEE PIMRC, 2016.

[5] P. Xiao, C. Toal, D. Burns, V. Fusco, and C. Cowan, "Transmit and receive filter design for OFDM based WLAN systems", in IEEE WCSP, 2010.

[6] R. Razavi, P. Xiao, and R. Tafazolli, "Information theoretic analysis of OFDM/OQAM with utilized intrinsic interference", IEEE Signal Processing Letters, vol. 22, no. 5, pp. 618-622, May 2015.

[7] J. Du, P. Xiao, J. Wu, and Q. Chen, "Design of isotropic orthogonal transform algorithm-based multicarrier systems with blind channel estimation", IET Communications, pp. 2695-2704, November 2012.

[8] L. Zhang, P. Xiao, A. Zafar, A. Quddus, and R. Tafazolli, "FBMC system: An insight analysis on double dispersive channel impact", IEEE Transactions on Vehicular Technology, 2016.

[9] M. Rodrigues and I. Darwazeh, "Fast OFDM: A proposal for doubling the data rate of OFDM schemes", IEEE/IEE International Conference on Telecommunications, 2002.

[10] W.H Chen, C. Smith, and S. Fralick, "A fast computational algorithm for the discrete cosine transform", IEEE Transactions on Communications, vol. 25, no. 9, pp. 1004-1009, Sep 1977.

[11] Zhongde Wang, "Fast algorithms for the discrete W transform and for the discrete fourier transform", IEEE Transactions on Acoustics, Speech, and Signal Processing, vol. 32, no. 4, pp. 803-816, Aug 1984.

[12] P. Tan and N. C. Beaulieu, "A comparison of DCT-based OFDM and DFT-based OFDM in frequency offset and fading channels", IEEE Transactions on Communications, pp. 2113-2125, Nov 2006.

[13] N. Al-Dhahir, H. Minn, and S. Satish, "Optimum DCT-based multicarrier transceivers for frequency-selective channels", IEEE Transactions on Communications, vol. 54, no. 4, pp. 760-760, April 2006.

[14] X. Ouyang and J. Zhao, "Single-tap equalization for fast OFDM signals under generic linear channels", IEEE Communications Letters, vol. 18, no. 8, pp. 1319-1322, Aug 2014.

[15] V. Sanchez, P. Garcia, A. M. Peinado, J. C. Segura, and A. J. Rubio, "Diagonalizing properties of the discrete cosine transforms", IEEE Transactions on Signal Processing, pp. 2631-2641, Nov 1995.

[16] J. Zhao and A. Ellis, "Transmission of 4-ASK optical fast OFDM with chromatic dispersion compensation", IEEE Photonics Technology Letters, vol. 24, no. 1, pp. 34-36, Jan 2012.

[17] G. D. Mandyam, "Sinusoidal transforms in OFDM systems", IEEE Transactions on Broadcasting, vol. 50, no. 2, pp. 172-184, June 2004.

[18] C. Y. Hung and W. H. Chung, "An improved MMSE-based MIMO detection using low-complexity constellation search", 2010 IEEE Globecom Workshops, pp. 746-750, Dec 2010.

[19] A. Trimeche, N. Boukid, A. Sakly, and A. Mtibaa, "Performance analysis of ZF and MMSE equalizers for mimo systems", 7th International Conference in Design Technology of Integrated Systems, pp. 1-6, May 2012.

[20] P. Xiao and R. Liu, "Multi-user detector for multi-carrier CDMA systems", Electronics Letters, pp. 1366-1368, November 2008.

[21] J Mao, M. Abdullahi, and P. Xiao, "A low complexity 256QAM soft demapper for 5G mobile system", EuCNC, 2016. 